Pacific Journal of Mathematics

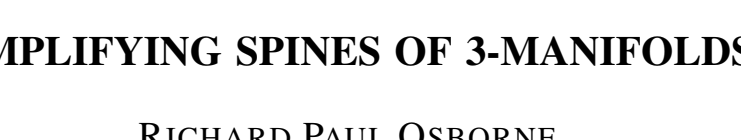




\title{
SIMPLIFYING SPINES OF 3-MANIFOLDS
}

\section{RICHARD OSBORNE}

\begin{abstract}
It is well known that every compact 3-manifold has a spine that is a 2-dimensional cell complex with just one vertex. Such a cell complex determines a group presentation in a natural way. It seems natural to call $K$ a simpler spine than $K^{\prime}$ if the presentation corresponding to $K$ is shorter than that corresponding to $K^{\prime}$. In this paper we give an algebraic condition which is sufficient to guarantee the existence of a simpler spine.
\end{abstract}

Of course, identifying the simplest spine of a 3-manifold would allow one to solve the homeomorphism problem for 3-manifolds. From one point of view the difficulty with identifying the simplest spines arises from the lack of correspondence between algebraic operations on presentations and geometric alteration of spines. For example $\left\langle a, b \mid a^{2} b^{3}, a^{3} b^{4}\right\rangle$ corresponds to a spine of $S^{3}$ but $\langle a, b| a^{2} b^{3} a^{3} b^{4}$, $\left.a^{3} b^{4}\right\rangle$ does not correspond to the spine of any 3-manifold. (See [7] for verification of this fact.) To state our result we need some definitions. Let $\phi=\langle X \mid \mathscr{R}\rangle$ be a group presentation, $X=\left\{x_{1}, x_{2}, \cdots, x_{n}\right\}$ and $\mathscr{R}=\left\{R_{1}, R_{2}, \cdots, R_{k}\right\} \mathscr{R}$ being a set of words in the free semigroup on $X \cup X^{-1}$. In what follows we will not distinguish between a relator $R_{i}$ and any cyclic conjugate of it or its inverse. This convention is adopted because the complexes determined are the same. Let $\phi^{\prime}=\left\langle X \mid R_{1}, R_{2}, \cdots, R_{i-1}, R_{i} R_{j}, R_{i+1}, \cdots, R_{k}\right\rangle$ where $j \neq i$. We shall say that $\phi^{\prime}$ was obtained from $\phi$ by multiplication of $R_{i}$ and $R_{j}$. If $\mathscr{A}$ is an automorphism of $F(X)$ (the free group on $X$ ) we denote by $\mathscr{A}\left(R_{i}\right)$ the image of $R_{i}$ under $\mathscr{A}$. We denote by $\mathscr{A}(\phi)$ the presentation $\left\langle X \mid \mathscr{A}\left(R_{1}\right), \cdots, \mathscr{A}\left(R_{k}\right)\right\rangle$. The length of a presentation $\phi$ is the sum of the lengths of the freely reduced relators of $\phi$.

THeOREM 1. If $K_{\phi}$ is the spine of a 3-manifold $M$ with corresponding presentation $\phi, \bar{\phi}$ is obtained from $\phi$ by automorphism or multiplication and the length of $\bar{\phi}$ is less than the length of $\dot{\phi}$ then $M$ has a simpler spine than $K_{\phi}$. This spine has a presentation $\phi^{\prime}$ that can be obtained from $\phi$ by automorphism and or by multiplication. One can assume that $\phi^{\prime}$ is at least as short as $\bar{\phi}$ if $\bar{\phi}$ was obtained from $\phi$ by automorphism or if $\phi$ could not be reduced in length by an automorphism.

A proof of this theorem appears in $\S 3$. Actually a somewhat stronger theorem is proved, as will be pointed out. We now give some examples to illustrate Theorem 1. 
EXAMPLE 1. $\phi=\left\langle a, b \mid a^{2} b^{2}, a^{3} b^{3} a^{3} b^{5}\right\rangle$ corresponds to a spine of a closed 3-manifold $M$ [7]. Furthermore $\phi$ cannot be reduced by an automorphism [9]. If we multiply a conjugate of the second relator by the inverse of the first relator and freely reduce we get $\bar{\phi}=\left\langle a, b \mid a^{2} b^{2}, a b a^{3} b^{5}\right\rangle$.

Theorem 1 guarantees that $M$ has a simpler spine than that corresponding to $\phi$. While $\bar{\phi}$ is not a presentation of such a spine we know that there is a spine of $M$ whose presentation is at least as short as $\bar{\phi}$. (Incidentally, $\phi$ presents the quaternions and uniquely determines the known closed manifold with this fundamental group [4].)

EXAMPLE 2. ${ }^{1} \phi=\left\langle a, b, c \mid a c^{-1} b a^{-1} c, a b a^{-1} b^{-1} c, a b^{-1} c^{-1} b c\right\rangle$ corresponds to a spine of a closed 3-manifold $M$. (Whose $R$ - $R$ system is shown in Figure 1.) There is an automorphism on $F(a, b, c)$ that

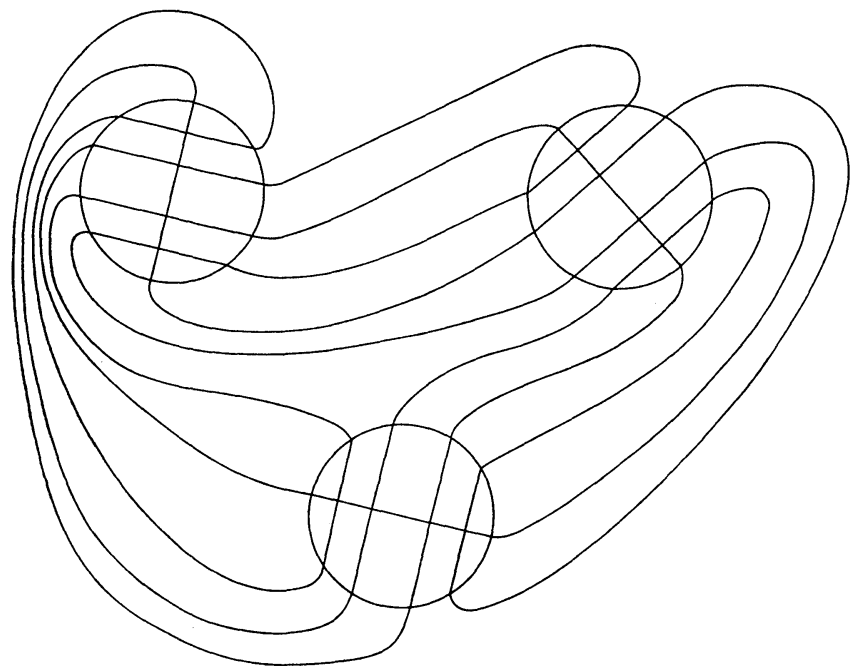

FIGURE 1

reduces the length of $\phi$, namely that defined by $a \rightarrow a, b \rightarrow b a, c \rightarrow c$. Now $\mathscr{A}(\phi)=\left\langle a, b, c \mid a c^{-1} b^{-1} c, b c, a b a^{-1} b^{-1} c b a c\right\rangle$. The length of $\mathscr{A}(\phi)$ is 14 , less than 15 which is the length of $\phi$. It follows that $M$ has a simpler spine than that corresponding to $\phi$. This presentation is interesting because the group presented has a trivial abelianization. One can easily do an elimination and get a 2-generator presentation $\left\langle b, c \mid c^{-2} b c b^{-1} c b, b^{-2} c b c^{-1} b c\right\rangle$. It is not known to this author if this group is trivial or not. This is the simplest possible spine for a counterexample to the Poincaré conjecture. One can show that $b^{5}=\left(c^{-1} b\right)^{5}$ and lies in the center of this group. Thus one can investigate

\footnotetext{
${ }^{1}$ Now known to be the binary dodecahedral space.
} 
$\left\langle b, c \mid c^{-2} b c b^{-1} c b, b^{-2} c b c^{-1} b c, b^{5},\left(c^{-1} b\right)^{5}\right\rangle$. This group is trivial if and only if the original group is trivial. If this group is indeed trivial then we have a very exciting example to investigate because the manifold $M$ survives every known method for showing that it is $S^{3}$.

EXAMPLE 3. $\phi=\left\langle a, b \mid a^{2} b^{3} a^{3} b^{3}, a^{2} b^{3} a^{2} b^{2}\right\rangle$ is the spine of a closed 3-manifold. Multiplying the first relator by the inverse of the second gives $\bar{\phi}=\left\langle a, b \mid a b, a^{2} b^{3} a^{2} b^{2}\right\rangle$. By Theorem 1 there is a geometrically allowable multiplication that results in at least as short a presentation as $\bar{\phi}$. This implies that $\bar{\phi}$ is actually a spine of $M$ since every multiplication gives this presentation or a longer one. Successive multiplications of the second relator by $(a b)^{-1}$ yield $b$. Again, if performed in the right order Theorem 1 implies these succesive presentations must correspond to spines of $M$. We see then that $M$ is $S^{3}$. This proof is purely algebraic in nature. Once a presentation of this spine is obtained one need never consult the geometry again to show that we have a spine of $S^{3}$.

2. Some powerful conjectures. In this section we present two group theoretic conjectures about presentations of spines which have very powerful consequences.

Definition 2.1. The presentation $\phi$ is said to reduce monotonical$l y$ to $\phi^{*}$ if there is a sequence of automorphisms and multiplications which monotonically reduce $\phi$ in length to $\phi^{*} . \phi$ has the monotone reduction property if every presentation $\phi^{\prime}$ to which $\phi$ reduces monotonically, itself reduces monotonically to the obviously trivial presentation $\left\langle x_{1}, x_{2}, \cdots, x_{n} \mid x_{1}, x_{2}, \cdots, x_{n}, 1,1, \cdots, 1\right\rangle$.

Conjecture 1. If $\phi$ corresponds to the spine of a 3-manifold and $\dot{\phi}$ reduces monotonically to the obviously trivial presentation, then $\phi$ has the monotone reduction property.

Conjecture 2. Conjecture 1 holds if we add the assumption that $\phi$ corresponds to the spine of a closed manifold.

Intuitively, these conjectures say that one cannot do the wrong reduction and get stuck when simplifying the spine of a simply connected 3-manifold.

THEOREM 2.1. If $K_{\phi}$ is a spine of $M$ and $\phi$ has the monotone reduction property then $M$ must be a cube with holes.

Proof. Since $\dot{\phi}$ can be reduced in length it follows from Theorem 1 that $M$ has a simpler spine than $K_{\phi}$. The resulting shorter 
presentation can again be reduced. Induction yields finally the obviously trivial presentation which can only correspond to a spine of a cube with holes.

Corollary 2.2. Conjecture 1 implies that the Poincaré conjecture is true.

Proof. In [3] Haken proves that a homotopy 3-sphere has a cell decomposition with one vertex whose 2 -skeleton $K_{\phi}$ has a presentation $\phi$ which can be monotonically reduced to the obviously trivial presentation.

If Conjecture 1 holds then Theorem 2.1 implies that a regular neighborhood of $K_{\phi}$ is a cube with holes. This in turn implies that the original manifold was $S^{3}$.

It is tempting to try to give a completely algebraic proof of Conjecture 1 , that is try to prove all group presentations that can be monotonically reduced to the obviously trivial presentation must have the monotone reduction property. That this cannot be done is shown by the presentation $\left\langle a, b \mid a^{-1} b a b^{-1} a b, \alpha^{2} b^{-1} a b a^{-1} b^{-1} a^{-1} b\right\rangle$. If we multiply the second relator by a conjugate of the inverse of the first relator we get $\left\langle a, b \mid a^{-1} b a b^{-1} a b, b^{-2} a^{-1} b a\right\rangle$. Now multiplying the first relator by the second we get $\left\langle a, b \mid a^{-1} b a^{2} b^{-1}, b^{-2} a^{-1} b a\right\rangle$. Now we multiply the first relator by a conjugate of the inverse of the second relator to get $\left\langle a, b \mid a b, b^{-2} a^{-1} b a\right\rangle$. This presentation easily reduces monotonically to $\langle a, b \mid a, b\rangle$. We now return to the original presentation and do another series of multiplications and automorphisms. First multiply as shown:

$$
\left(a b^{-1} a b a^{-1} b\right)\left(b^{-1} a^{-1} b a^{2} b^{-1} a b a^{-1}\right)=a b^{-1} a b a^{-2} b a^{2} b^{-1} a b a^{-1}
$$

which cyclically reduces to $a^{2} b a^{-2} b a^{2} b^{-1}$. Our presentation is now

$$
\left\langle a, b \mid a^{-1} b a b^{-1} a b, a^{2} b a^{-2} b a^{2} b^{-1}\right\rangle \text {. }
$$

Now we do the automorphism defined by $a \rightarrow a$ and $b \rightarrow a b^{-1}$ to get $\left\langle a, b \mid a b a b^{-2}, a^{2} b^{-1} a^{-1} b^{-1} a^{2} b\right\rangle$. We now multiply the second relator by the first to get $\left\langle a, b \mid a b a b^{-2}, a b^{2} a b^{-1} a^{-1} b^{-1}\right\rangle$. Now we do the automorphism defined by $a \rightarrow a b^{-1}$ and $b \rightarrow b$. We get $\left\langle a, b \mid a^{2} b^{-3}, a b a b^{-1} a^{-1} b^{-1}\right\rangle$. It is easy to check that this presentation cannot be reduced further by automorphism or multiplication. The above presentation gives a counterexample for Conjectures 2 and 4 of [1]. None of the above presentations correspond to a spine of a 3-manifold, hence do not provide counterexamples for our Conjectures 1 or 2 .

THEOREM 2.3. Conjecture 2 implies that monotone reduction of the presentation of a spine gives an algorithmic method for identify- 
ing $S^{3}$ among compact 3-complexes. The proof of this theorem is long and somewhat involved and will be presented in a subsequent paper.

Connections between transformations of group presentations and manifolds have a long history. Whitehead studied multiplications and automorphisms in [9]. In [2] Andrews and Curtis discuss changing presentations by what amounts to sequences of multiplications and their relations with 4-manifolds. In [5] the author relates these to the 3- and 4-dimensional Poincaré conjecture. Rapaport [8] shows that presentations of the trivial group which can be reduced to the obviously trivial presentation by automorphism and multiplication can be reduced by multiplication alone. This process does not preserve montone reduction, however.

3. Proof of Theorem 1. If $\phi$ can be reduced in length by an automorphism then Theorem 1 of [10] can be applied to show that tnere is a geometrically allowable transformation which yields at least as short a presentation. (See also [6] for the connection between Zieschang's theorem and presentations of spines.) We now assume that the manifold $M$ has a spine $K_{\phi}$ whose corresponding presentation $\phi$ cannot be reduced by automorphism. Let us assume that $\phi=\left\langle x_{1}, \cdots, x_{n} \mid R_{1}, \cdots, R_{k}\right\rangle$ and that

$$
\bar{\phi}=\left\langle x_{1}, \cdots, x_{n} \mid R_{1} R_{2}, R_{2}, \cdots, R_{k}\right\rangle
$$

is shorter than $\phi$ when the free cancellations in $R_{1} R_{2}$ are done. Let us further suppose that $R_{1}=U x_{i_{1}}^{n_{1}} x_{i_{2}}^{n_{2}} \cdots x_{i_{r}}^{n_{r}}$ and

$$
R_{2}=x_{i_{r}}^{-n_{r}} x_{i_{r-1}}^{-n_{r-1}} \cdots x_{i_{1}}^{-n_{1}} V
$$

where $U$ and $V$ are words on $x_{1}, x_{2}, \cdots, x_{n}$ and $U V$ is a cyclically reduced word. Since $R_{1} R_{2}$ is assumed to be shorter than $R_{1}$ it must be that the length of $V$ is less than one half the length of $R_{2}$.

Now we examine the (reduced) $R-R$ system corresponding to

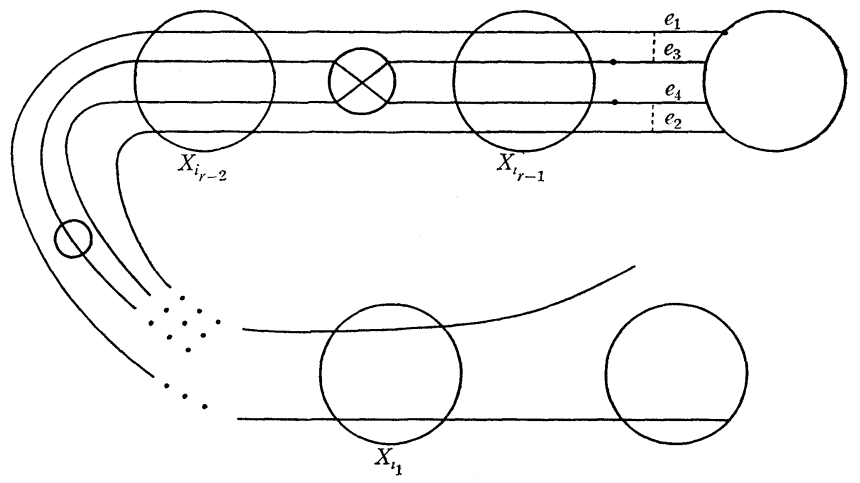

Figure 2 
the faithfully embedded $P$-graph of $K_{\phi}$. In what follows we shall assume that the reader is familiar with $R-R$ systems and operations on them as developed in [6]. We locate on this $R-R$ system the route $e_{1}$ of $R_{1}$ corresponding to the gap between $x_{i_{r-1}}^{n} r_{r-1}$ and $x_{i_{r}}^{n}$ and also the route $e_{2}$ of $R_{2}$ corresponding to the gap between $x_{i_{r-1}}^{-n_{r-1}}$ and $x_{i_{r}}^{-n_{r}}$. (See Figure 2.) Now if we could always geometrically perform the multiplication between these two routes then the theorem would be proved. However we cannot always do this multiplication geometrically because there may be intervening routes. We shall find a different place to multiply geometrically that results in at least as much free cancellation as resulted between $R_{1}$ and $R_{2}$. We assume without loss of generality that an end of $e_{2}$ lies clockwise from an end of $e_{1}$ in town $x_{i_{r-1}}$. Beginning at $e_{2}$ we proceed counterclockwise until we encounter the first route not lying in $R_{2}$. Call this route $e_{3}$ and the corresponding relator $R_{3}$. (Note that $R_{3}=R_{2}$ is possible.) Denote by $e_{4}$ the first clockwise route from $e_{3}$. Of necessity $e_{4}$ is a route of $R_{2}$. We multiply $R_{2}$ and $R_{3}$ between $e_{4}$ and $e_{3}$. The idea of the proof that

$$
\phi^{\prime}=\left\langle x_{1}, \cdots, x_{n} \mid R_{1}, R_{2}, R_{3} R_{2}, R_{4}, \cdots, R_{k}\right\rangle
$$

is as short as $\bar{\phi}$ is to argue that because $e_{3}$ and $e_{4}$ are "caugh between" $e_{1}$ and $e_{2}$ the words $R_{2}$ and $R_{3}$ must be parallel (go through the same stations) at least as long as $R_{1}$ and $R_{2}$. We refer to the region in which the edges of $R_{1}$ and $R_{2}$ pass through the same stations, where the syllables $x_{i_{1}}^{n_{1}}, \cdots, x_{i_{r}}^{n_{r}}$ appear, as the free cancellation region. However, there are a few things that could go wrong with this argument. One of these is shown in Figure 2. As shown, there may be one or several towns in the free cancellation region so that the tracks of $R_{2}$ and $R_{3}$ do not remain parallel in this region. In this case we may remove all such towns from the free cancellation region by pulling them through the towns $x_{i_{r-1}}, x_{i_{r-2}}, \cdots, x_{i_{1}}$ along tracks parallel to the tracks of $R_{1}$ and $R_{2}$. This process amounts to an automorphism of $\phi$. This automorphism does not change the length of the presentation. For if it did then pulling one of these

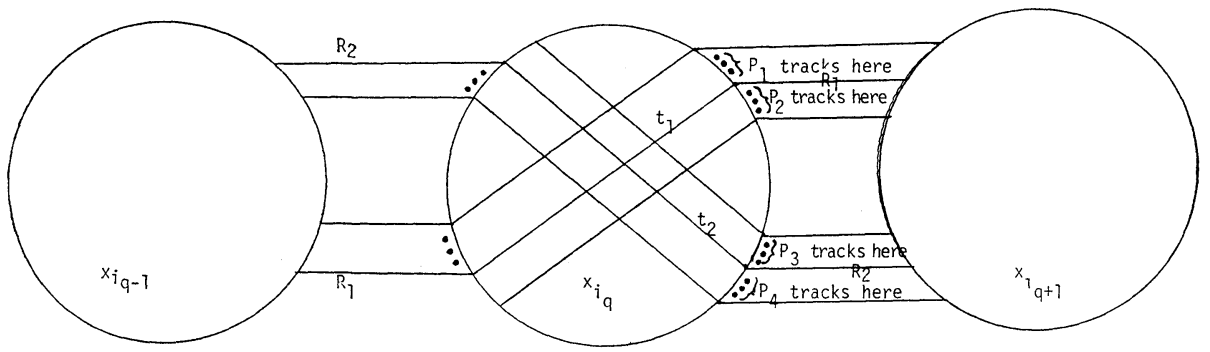

FIGURE 3 
towns in the opposite direction would decrease the length by an automorphism, contradicting our hypothesis. There is one more type of difficulty which might arise. This is illustrated in Figure 3. This difficulty occurs when there is a cross of the supposedly parallel tracks of $R_{1}$ and $R_{2}$ somewhere in the free cancellation region. Since the corresponding syllables must be the same length it follows that they must both be length 1 or -1 since crossing syllables must have relatively prime length. Assume without loss of generality that both syllables have length 1 . Let us assume that the cross appears in the town $x_{i_{q}}$ with tracks $t_{1}$ and $t_{2}$ crossing, $t_{1}$ lying in $R_{1}, t_{2}$ in $R_{2}$. We shall further assume that there are: $p_{1}$ tracks in town $x_{i_{q}}$ lying countercrockwise from $t_{1}$ in the same station as $t_{1} ; p_{2}$ tracks in town $x_{i_{q}}$ lying clockwise from $t_{1}$ in the same station as $t_{1} ; p_{3}$ tracks lying counterclockwise from $t_{2}$ in the same station as $t_{2}$; and $p_{4}$ tracks in the sams station as $t_{2}$ lying clockwise from $t_{2}$. These assumptions imply that there are: $p_{1}+p_{4}$ pairs of syllables of the form $\left(x_{i_{q-1}}^{n q-1} x_{i_{q}}\right)^{ \pm 1} ; p_{2}+p_{3}$ pairs of syllables of the form $\left(x_{i_{q}} x_{i_{q+1}}^{n}\right)^{ \pm 1}$ in $\phi$; and the two occurences of syllables of the form, $\left(x_{i_{q-1}}^{n_{q}-1} x_{i_{q}} x_{i_{q+1}}^{n_{q+1}}\right)^{ \pm 1}$ not counted above. All occurences of $x_{i_{q}}$ must be in one of the above forms or in the form $\left(x_{i_{q-1}}^{n q-1} x_{i_{q}}^{2} x_{i_{q+1}}^{n}\right)^{ \pm+1}$. If $p_{2}+p_{3}+2>p_{1}+p_{4}$ then the automorphism $x_{i_{q}} \rightarrow x_{i_{q}} x_{i_{q+1}}^{-1}$ and $x_{j} \rightarrow x_{j}$ for $j \neq i_{q+1}$ reduces the length of $\phi$ contrary to hypothesis. If $p_{2}+p_{3} \leqq p_{1}+p_{4}$ then $x_{i_{q}} \rightarrow x_{\imath_{q-1}}^{-1} x_{\imath_{q}}$ and $x_{j} \rightarrow x_{j}$ for $j \neq i_{q-1}$ defines an automorphism of $\dot{\phi}$ that decreases its length. From the above argument we see that a cross cannot occur in the free cancellation region. A cross may occur at the ends of this region but this presents no problem since all free cancellations may be (geometrically) performed. This completes the proof.

\section{REFERENCES}

1. J. J. Andrews and M. L. Curtis, Extended Nielsen operations in free groups, Amer. Math. Monthly, 73 (1966), 21-28.

2. - Free groups and handlebodies, Proc. Amer. Math. Soc., 16 (1965), 192-195.

3. W. Haken, Algebraically trivial decompositions of homotopy 3-spheres, Illinois J. Math., 12 (1966), 133-170.

4. J. Milnor, Groups which act on $S^{n}$ without fixed points, Amer. J. Math., 79 (1957), 623-630.

5. R. P. Osborne, On the 4-dimensional Poincaré conjecture for manifolds with 2dimensional spines, Canad. Math. Bull., to appear.

6. R. P. Osborne and R. S. Stevens, Group presentations corresponding to spines of 3manifolds $I I$, preprint.

7. R. P. Osborne, The simplest closed 3-manifolds, Pacific J. Math., to appear.

8. E. S. Rapaport, Groups of order 1; Some properiies of presentations, Acta Math., 121 (1968), 127-150.

9. J. H. C. Whitehead, On equivalent sets of elements in a free group, Ann. Math.,

(2) 37 (1936), 782-800. 
10. H. Zieschang, On Simple systems of paths on complete pretzels, Transl. Amer. Math. Soc., (2) 92 (1970), 127-137.

Received May 3, 1976 and in revised form August 13, 1976.

Colorado State University

FORT COLLINS, CO 80523 


\section{PACIFIC JOURNAL OF MATHEMATICS}

\section{EDITORS}

RICHARD ARENS (Managing Editor)

University of California

Los Angeles, CA 90024

Charles W. Curtis

University of Oregon

Eugene, OR 97403

C. C. Moore

University of California

Berkeley, CA 94720

\section{J. DugundJI}

Department of Mathematics

University of Southern California

Los Angeles, CA 90007

R. FInN and J. Milgram

Stanford University

Stanford, CA 94305

\section{ASSOCIATE EDITORS}
E. F. BECKENBACH
B. H. NeumanN
F. WOLF
K. YoSHIDA

\section{SUPPORTING INSTITUTIONS}

\author{
UNIVERSITY OF BRITISH COLUMBIA \\ CALIFORNIA INSTITUTE OF TECHNOLOGY \\ UNIVERSITY OF CALIFORNIA \\ MONTANA STATE UNIVERSITY \\ UNIVERSITY OF NEVADA, RENO \\ NEW MEXICO STATE UNIVERSITY \\ OREGON STATE UNIVERSITY \\ UNIVERSITY OF OREGON
}

\author{
UNIVERSITY OF SOUTHERN CALIFORNIA \\ STANFORD UNIVERSITY \\ UNIVERSITY OF HAWAII \\ UNIVERSITY OF TOKYO \\ UNIVERSITY OF UTAH \\ WASHINGTON STATE UNIVERSITY \\ UNIVERSITY OF WASHINGTON
}

The Supporting Institutions listed above contribute to the cost of publication of this Journal, but they are not owners or publishers and have no responsibility for its content or policies.

Mathematical papers intended for publication in the Pacific Journal of Mathematics should be in typed form or offset-reproduced, (not dittoed), double spaced with large margins. Please do not use built up fractions in the text of the manuscript. However, you may use them in the displayed equations. Underline Greek letters in red, German in green, and script in blue. The first paragraph or two must be capable of being used separately as a synopsis of the entire paper. Items of the bibliography should not be cited there unless absolutely necessary, in which case they must be identified by author and journal, rather than by item number. Manuscripts, in triplicate, may be sent to any one of the editors. Please classify according to the scheme of Math. Reviews, Index to Vol. 39. All other communications should be addressed to the managing editor, or Elaine Barth, University of California, Los Angeles, California, 90024.

50 reprints to each author are provided free for each article, only if page charges have been substantially paid. Additional copies may be obtained at cost in multiples of 50 .

The Pacific Journal of Mathematics is issued monthly as of January 1966. Regular subscription rate: $\$ 72.00$ a year (6 Vols., 12 issues). Special rate: $\$ 36.00$ a year to individual members of supporting institutions.

Subscriptions, orders for numbers issued in the last three calendar years, and changes of address should be sent to Pacific Journal of Mathematics, 103 Highland Boulevard, Berkeley, California, 94708. Older back numbers obtainable from Kraus Periodicals Co., Route 100, Millwood, NY 10546.

PUBLISHED BY PACIFIC JOURNAL OF MATHEMATICS, A NON-PROFIT CORPORATION

Printed at Kokusai Bunken Insatsusha (International Academic Printing Co., Ltd.). 8-8, 3-chome, Takadanobaba, Shinjuku-ku, Tokyo 160, Japan.

Copyright (C) 1978 by Pacific Journal of Mathematics

Manufactured and first issued in Japan 


\section{Pacific Journal of Mathematics

Vol. 74, No. $2 \quad$ June, 1978

Aharon Atzmon, Spectral synthesis in some spaces of bounded continuous

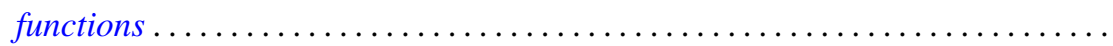

Karl Egil Aubert and Isidor Fleischer, Tensor products of ideal systems and their modules.............................................

Richard F. Basener, Several dimensional properties of the spectrum of a uniform

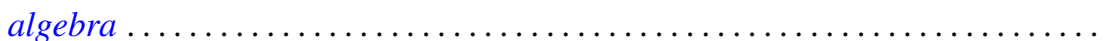

R. H. Bing and Michael Peter Starbird, Super triangulations ............. 307

Andrew Carson, Coherent polynomial rings over regular rings of finite index .....

Robert M. DeVos and Frederick W. Hartmann, Sequences of bounded summability domains .................................................

George Grätzer and R. Padmanabhan, Symmetric difference in abelian groups ....

Robert L. Griess, Jr., A remark about groups of characteristic 2-type and

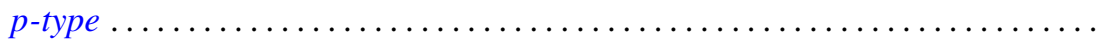

Emil Grosswald and F. J. Schnitzer, A class of modified $\zeta$ and L-functions........

Jutta Hausen and Johnny Albert Johnson, Ideals and radicals of some

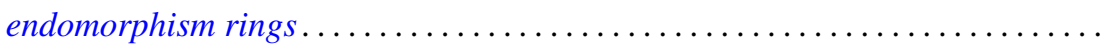

Jean Ann Larson, A solution for scattered order types of a problem of Hagendorf ............................................

Peter A. McCoy, Extremal properties of real biaxially symmetric potentials in $E^{2(\alpha+\beta+2)}$

Héctor Alfredo Merklen, Hereditary crossed product orders .

Hal G. Moore and Adil Mohamed Yaqub, Equational definability of addition in certain rings...

Robert Laurens Moore, Reductivity in $C^{*}$-algebras and essentially reductive operators. . .

Joseph Alvin Neisendorfer, Lie algebras, coalgebras and rational homotopy theory for nilpotent spaces...

William Raymond Nico, Bounded monoids

Richard Paul Osborne, Simplifying spines of 3-manifolds ...

Richard Paul Osborne, The simplest closed 3-manifolds. With an appendix by Osborne and J. Yelle.

Clayton Collier Sherman, The $K$-theory of an equicharacteristic discrete valuation ring injects into the $K$-theory of its field of quotients.... .

Mitchell Herbert Taibleson, The failure of even conjugate characterizations of $H^{1}$

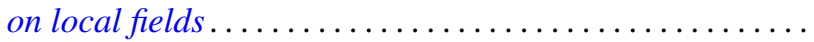

Keti Tenenblat, On characteristic hypersurfaces of submanifolds in Euclidean space ...................................

Jeffrey L. Tollefson, Involutions of Seifert fiber spaces..........

Joel Larry Weiner, An inequality involving the length, curvature, and torsions of a curve in Euclidean $n$-space .......................

Neyamat Zaheer, On generalized polars of the product of abstract homogeneous polynomials.... 